\title{
ALICE SAMPA-ASIC Second-Prototype Qualification Studies for LHC Run 3 and Beyond
}

\author{
G. Tambave*i \\ On behalf of the ALICE Collaboration \\ Department of Physics and Technology, University of Bergen, Norway \\ E-mail: Ganesh. Tambave@uib.no
}

\begin{abstract}
ALICE experiment studies the properties of the Quark-Gluon Plasma a new state of matter which is expected to be created at the high-energy densities reached at the CERNs LHC. In 2019 after the long-shutdown of LHC, a major upgrade of the ALICE sub-detectors is planned. The ALICE TPC is one of them which will be upgraded from wire chambers to gas electron multiplier chambers and the new TPC will acquire data in continuous-readout mode. In this context, a new frontend ASIC named SAMPA is being developed. This paper reports the outcome of the SAMPA characterization tests carried out using a pulse generator and a prototype GEM chamber.
\end{abstract}

Topical Workshop on Electronics for Particle Physics

11 - 14 September 2017

Santa Cruz, California

\footnotetext{
* Speaker.

$\dagger$ Author would like to thank Dezso Varga, Wigner Institute Budapest for GEM chamber design, SAMPA design team, Sao Paulo/Bergen, SAMPA carrier board design team, IPNO Orsay and Christian Lippmann, GSI Darmstadt for the help and support during this work.
} 


\section{Introduction}

A Large Ion Collider Experiment (ALICE) [1] is a general purpose heavy-ion ( $\mathrm{Pb}-\mathrm{Pb})$ experiment ongoing at the CERNs LHC. It is designed to study the physics of strongly interacting matter and quark-gluon plasma at high-energy densities and temperature. Currently, by design the ALICE detector system is capable to handle $\mathrm{Pb}-\mathrm{Pb}$ interaction rate of few kHz. However, in 2021 after the second long-shutdown (LS2) of the LHC the expected $\mathrm{Pb}-\mathrm{Pb}$ interaction rate is about $50 \mathrm{kHz}$. To handle the increased interaction rate a major upgrade of the ALICE sub-detectors will take place during the LS2.

In the planned upgrade, the ALICE TPC [1] will make use of high-rate capable readout chambers based on Gas Electron Multiplier (GEM) technology [2] and in addition, the readout electronics will acquire data in a continuous mode. To achieve the continuous readout, a newly designed front-end ASIC named SAMPA [1] will process the signals from the GEM pads. The output data from the SAMPA will be transferred using Gigabit transfer links [3] to common readout unit [1] where the necessary data-processing for the physics analysis will be done.

\section{SAMPA ASIC}

The SAMPA ASIC is being developed as a part of ALICE upgrade project. The SAMPA contains the front-end electronics designed to readout the GEM chamber pads of the upgraded ALICE TPC. The SAMPA will also be used in the upgraded ALICE Muon chambers [1]. The SAMPA combines functionality of the previous PASA [4] and ALTRO [5] chips into one ASIC. It is capable to handle bipolar input signals over 32 channels running in continuous readout mode. The SAMPA contains Charge Sensitive pre-Amplifiers (CSAs), pulse shapers, Analogue-to-Digital Converters (ADCs) and a Digital signal Processing (DSP) module as shown in Figure 1. The acquired data from the SAMPA can be transferred at rate 3.2 Gbps over 11 serial links.

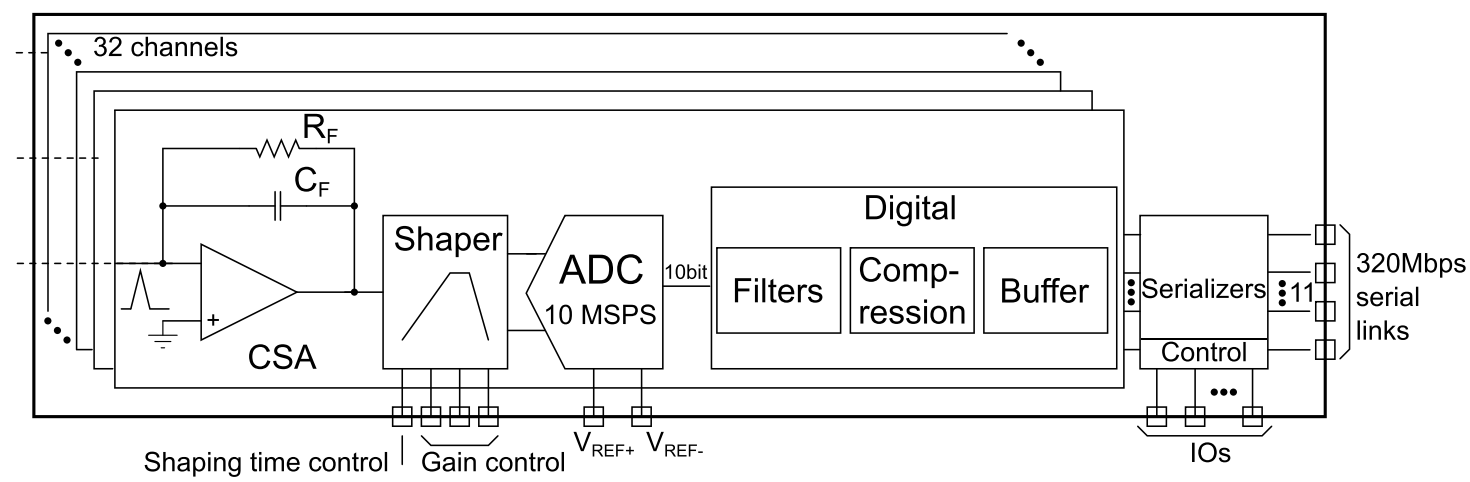

Figure 1: A block diagram of the SAMPA ASIC consisting of Charge-Sensitive preAmplifier (CSA), Shaper, Analogue-to-Digital Converter (ADC) and Digital Signal Processing (DSP) block made up of different data filters. 


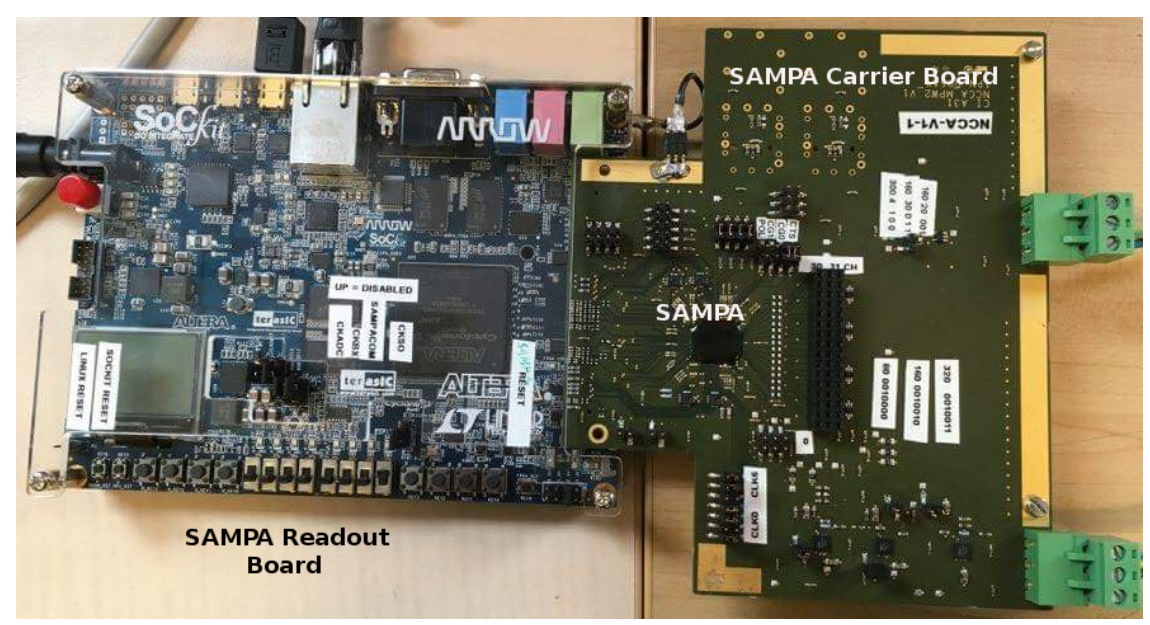

Figure 2: The SAMPA ASIC (mounted on carried-board) coupled to its readout-board. The SAMPA carrier board dimensions are about $15 \times 15 \mathrm{~cm}^{2}$.

\begin{tabular}{ccccc}
\hline $\begin{array}{c}\text { E-link speed } \\
{[\mathrm{MHz}]}\end{array}$ & $\begin{array}{c}\text { Sampling rate } \\
{[\mathrm{MHz}]}\end{array}$ & $\begin{array}{c}\text { Current } \\
{[\mathrm{mA}]}\end{array}$ & $\begin{array}{c}\text { Power } \\
\text { per 32 channels }[\mathrm{mW}]\end{array}$ & $\begin{array}{c}\text { Power } \\
\text { per channel }[\mathrm{mW}]\end{array}$ \\
\hline 080 & 10 & 277 & 346 & 11 \\
160 & 10 & 331 & 414 & 13 \\
320 & 10 & 436 & 545 & 17 \\
320 & 20 & 480 & 600 & 19 \\
320 & 05 & 307 & 384 & 12 \\
\hline
\end{tabular}

Table 1: The power consumption of the SAMPA operated at $1.25 \mathrm{~V}$ for the combination of different seriallink and sampling speeds, respectively. The consumed power is well below the requirement of $35 \mathrm{~mW} / \mathrm{ch}$.

\section{Characterization studies}

The first prototype (MPW1) of the SAMPA with three-channels was produced and tested during 2014-2015. The performance results of the MPW1 and its readout system are reported in [6, 7]. The ALICE TPC requirements from the SAMPA are as follows; Power consumption less than $35 \mathrm{~mW}$ per channel, Gain $20 \mathrm{mV} / \mathrm{fC}$ and $30 \mathrm{mV} / \mathrm{fC}$, Noise less than $800 \mathrm{ENC}$ at $22 \mathrm{pF}$ capacitance, 160 ns peaking time with $70 \Omega$ ESD protection resistance and cross-talk less than $0.3 \%$.

Most of these requirements were met by the MPW1 however, the noise and the cross-talk was higher than required. To improve the noise and the cross-talk performance, several improvements were implemented in the design of the second prototype. The improvements includes increase in gain-bandwidth product of CSA amplifier, use of short bond-wires and increase in gate-source voltage to reduce the thermal noise of the bias circuit. A photograph of the second prototype (mounted on carrier-board) coupled to its readout-board is shown in figure 2. In this section performance studies of the second prototype of SAMPA are reported.

The power consumption listed in table 1 is measured at $1.25 \mathrm{~V}$ for different serial-link-speed combined with sampling-frequency of the SAMPA ADC. The highest power $(19 \mathrm{~mW} / \mathrm{ch}) \mathrm{con}-$ sumed for the combination of $320 \mathrm{MHz}$ e-link speed and $20 \mathrm{MHz}$ sampling frequency is much better than the requirement $(<35 \mathrm{~mW} / \mathrm{ch})$. The ALICE TPC plans to operate at $160 \mathrm{MHz}$ e-link 

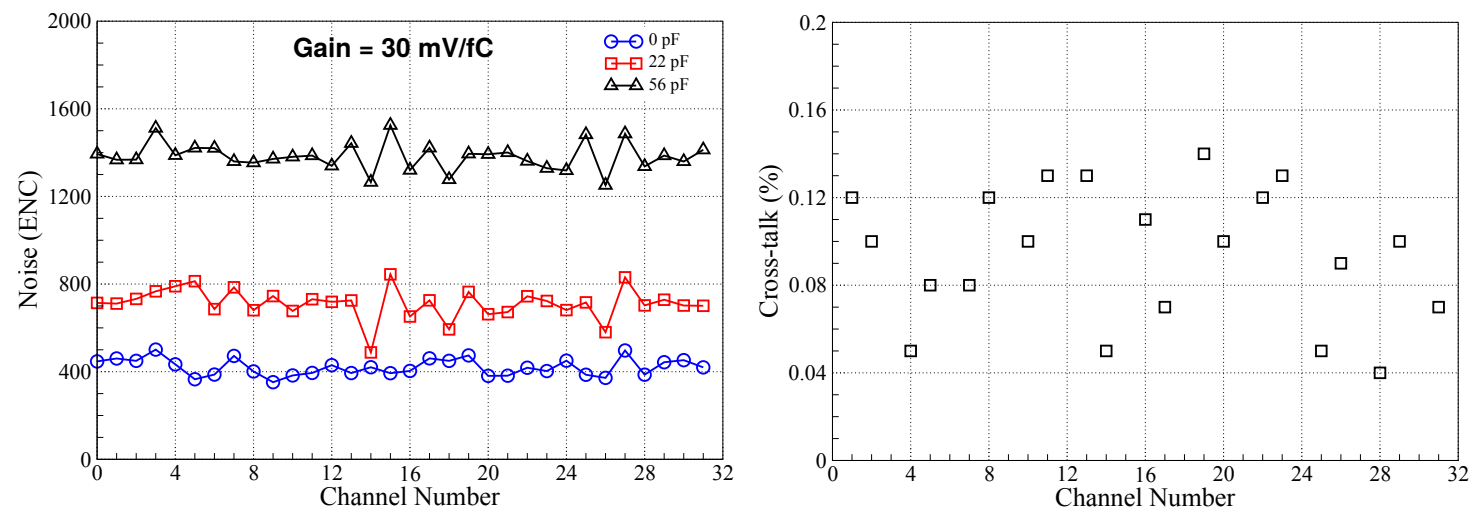

Figure 3: (Left) The SAMPA noise versus channel number for different input capacitances measured at $30 \mathrm{mV} / \mathrm{fC}$ gain and $160 \mathrm{~ns}$ peaking time. (Right) The cross-talk percentage versus channel number. Both requirement i.e. the noise less than $800 \mathrm{ENC}$ at $22 \mathrm{pF}$ capacitance and the cross-talk less than $0.3 \%$ are achieved.
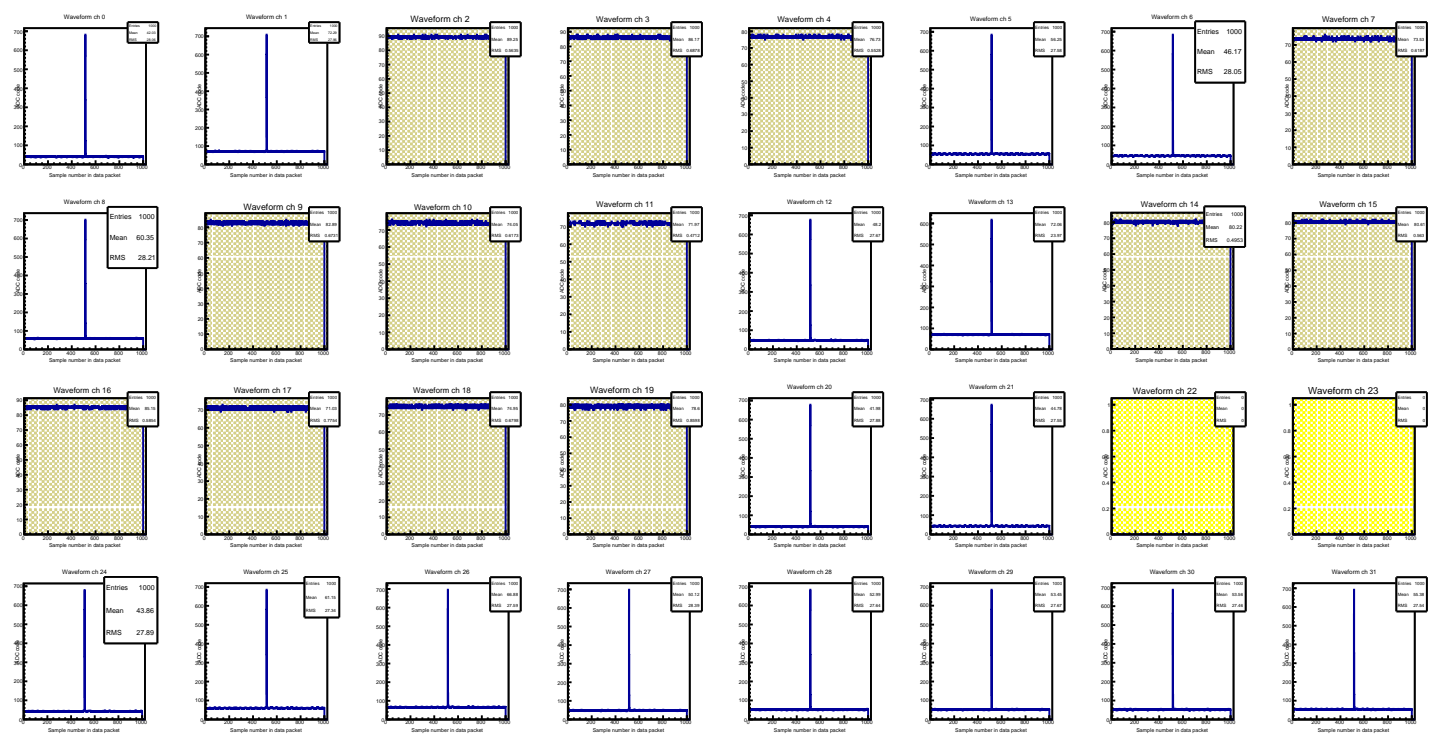

Figure 4: The 32 output waveforms of the destroyed SAMPA after the spark test. The completely destroyed channels are the empty windows filled with yellow color while a partly destroyed channels are filled with light yellow color where only signal baseline is visible and no impulse response as compared to the remaining channels.

speed and $5 \mathrm{MHz}$ sampling frequency. The SAMPA noise as function of channel number for three different capacitances is shown in figure 3 (Left). The noise is obtained at $30 \mathrm{mV} / \mathrm{fC}$ gain and $160 \mathrm{~ns}$ peaking time. The ALICE TPC requirement of noise less than $800 \mathrm{ENC}$ at $22 \mathrm{pF}$ is achieved.

The cross-talk percentage versus channel number is shown in figure 3 (Right). To obtained the cross-talk a highest possible test-charge is injected into every third input channel $(0,3,6 .$.$) of$ the SAMPA and the signal amplitude is measured in the neighbouring channels. The measured cross-talk percentage is well within the ALICE TPC requirement of $0.3 \%$. 
An Input Protection Scheme (IPS) is in place to protect the front-end of the SAMPA from discharges in the GEM-chamber. These discharges are expected to occur when a highly-ionising particle will hit the gaseous volume of the GEM-based ALICE TPC. The IPS is implemented inside the ASIC as well as on the carrier board of the SAMPA. The IPS consists of a series resistance and two NUP4114 ESD protection diodes. The value of series resistance for the IPS inside the ASIC was set to $5 \Omega$ while the outer/external resistance $\left(R_{o}\right)$ value was unknown. To optimize its value a simple experiment was carried out where discharges were created inside the GEM chamber operating at very high voltage $(4.2 \mathrm{KV})$ by irradiating it with alpha particles. Different values of $R_{o}(100 \Omega, 75 \Omega, 50 \Omega$, and $25 \Omega$ ) were used one-by-one while thousands of discharges were taking place inside the GEM chamber. The discharge rate was about 30 discharges-per min. It is observed that the front-end of the SAMPA was destroyed while $R_{o}$ til $50 \Omega$ was in place. The current drawn by the destroyed SAMPA went up to $1.5 \mathrm{~A}$ from $0.5 \mathrm{~A}$. As an example output of all 32 channels of the destroyed SAMPA are shown in figure 4. In the figure the empty windows (filled with yellow color) are completely destroyed (shorted to ground) channels while the windows filled with light yellow color shows partly destroyed channels with only baseline visible and not the impulse response as seen in the remaining channels. The SAMPA has survived almost 2400 discharges with $R_{o}$ of $70 \Omega$. Moreover, minor effect of $R_{o}$ on SAMPA noise is observed. Therefore, it would be worth to use $R_{o}$ of $70 \Omega$ for the protection of the SAMPA front-end from the GEM discharges.

\section{Conclusions}

The second prototype of the SAMPA ASIC has been produced and tested for the ALICE TPC upgrade planned during the Long shutdown of the LHC. As compared to the first prototype, all the set requirements for the upgrade are achieved by the second prototype and there is a significant improvement in the noise ( $<800 \mathrm{ENC}$ at $22 \mathrm{pF}$ capacitance, $160 \mathrm{~ns}$ peaking time) and cross-talk $(<0.3 \%)$ of the SAMPA. To protect the front-end of the SAMPA from the GEM discharges, an input protection scheme is implemented. This scheme has been tested for thousands of GEM discharges. The discharge test revealed that it is important to have series resistance value of $70 \Omega$ to protect the SAMPA front-end while keeping the required noise performance.

\section{References}

[1] The ALICE Collaboration, Upgrade of the ALICE Read-Out and Trigger System, Technical Design Report, CERN-LHCC-2013-019 (ALICE-TDR-015).

[2] F. Sauli, The GEM: Operating principles and applications, NIMA 805 (2016) 2-24.

[3] P. Moreira, The GBT-SerDes ASIC prototype, JINST 5 (2010) C11022.

[4] H. Soltveit et al., The pre-amp shaper for the ALICE TPC detector, NIMA, 676 (2012) 106-119.

[5] R. Bosch et al., The ALTRO chip: A 16-channel A/D converter and digital processor for gas detectors, IEEE TNS $\mathbf{5 0}$ (2003) no.6, 2460-2469.

[6] G. Tambave et al., Characterization of the first prototype of the ALICE SAMPA ASIC for LHC Run 3 and Beyond, JINST 12 (2017) C03012.

[7] G. Tambave and A. Velure, Qualification of the ALICE SAMPA ASIC with a High-Speed Continuous DAQ System, IEEE-TNS 64 (2017) no.6, 1461. 